Erratum

\title{
Erratum to "Effect of Telmisartan or Losartan for Treatment of Nonalcoholic Fatty Liver Disease: Fatty Liver Protection Trial by Telmisartan or Losartan Study (FANTASY)"
}

\author{
Takumi Hirata, ${ }^{1}$ Kengo Tomita, ${ }^{1,2}$ Toshihide Kawai, ${ }^{1}$ Hirokazu Yokoyama, \\ Akira Shimada, ${ }_{1}^{1}$ Masahiro Kikuchi, ${ }^{1}$ Hiroshi Hirose, ${ }^{1}$ Hirotoshi Ebinuma, ${ }^{1}$ Junichiro Irie, ${ }^{1}$ \\ Keisuke Ojiro, ${ }^{1}$ Yoichi Oikawa, ${ }^{1}$ Hidetsugu Saito, ${ }^{1}$ Hiroshi Itoh, ${ }^{1}$ and Toshifumi Hibi ${ }^{1}$ \\ ${ }^{1}$ Department of Internal Medicine, School of Medicine, Keio University, 35 Shinanomachi, Shinjuku-ku, Tokyo 160-8582, Japan \\ ${ }^{2}$ Department of Internal Medicine, National Defense Medical College, 3-2 Namiki, Tokorozawa-Shi, Saitama 359-8513, Japan \\ Correspondence should be addressed to Toshihide Kawai; tkawai@a3.keio.jp \\ Received 5 March 2014; Accepted 16 April 2014; Published 27 April 2014 \\ Copyright (C) 2014 Takumi Hirata et al. This is an open access article distributed under the Creative Commons Attribution License, \\ which permits unrestricted use, distribution, and reproduction in any medium, provided the original work is properly cited.
}

In the paper, the dosage of telmisartan is incorrect due to a typographical error. Twelve patients, assigned to telmisartan group, received telmisartan at a dose of $40 \mathrm{mg}$, not $20 \mathrm{mg}$, once a day. The correct sentences should appear as given below.

(1) "Nineteen hypertensive NAFLD patients with type 2 diabetes were randomly assigned to receive telmisartan at a dose of $40 \mathrm{mg}$ once a day $(n=12)$ or losartan at a dose of $50 \mathrm{mg}$ once a day $(n=7)$ for 12 months" in the "Methods" section of the "Abstract."

(2) "Nineteen hypertensive NAFLD patients with type 2 diabetes were randomly assigned to the telmisartan (T) group (receiving a standard dose of $40 \mathrm{mg}$ once daily, $n=12$ ) or the losartan (L) group (receiving a standard dose of $50 \mathrm{mg}$ once daily, $n=7$ )" in Section 2.2 . 


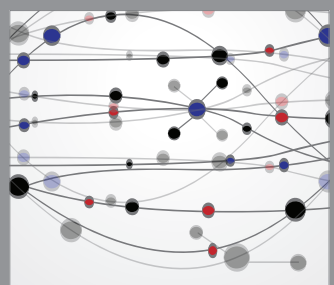

The Scientific World Journal
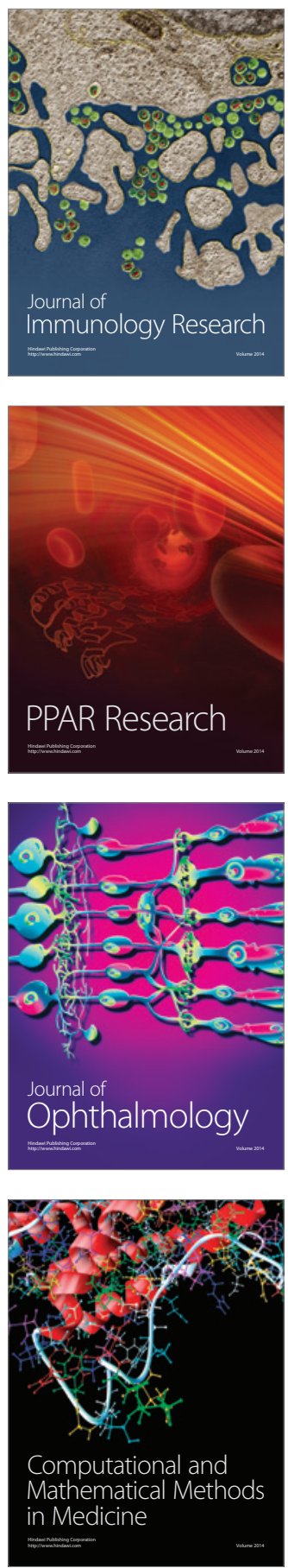

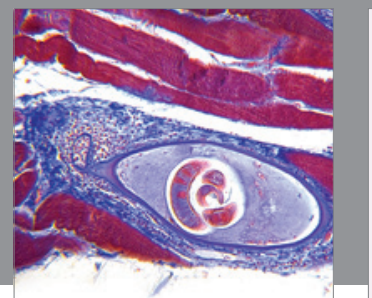

Gastroenterology

Research and Practice
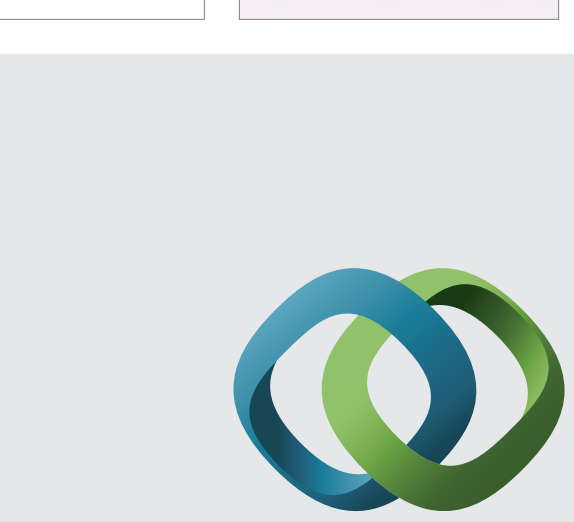

\section{Hindawi}

Submit your manuscripts at

http://www.hindawi.com

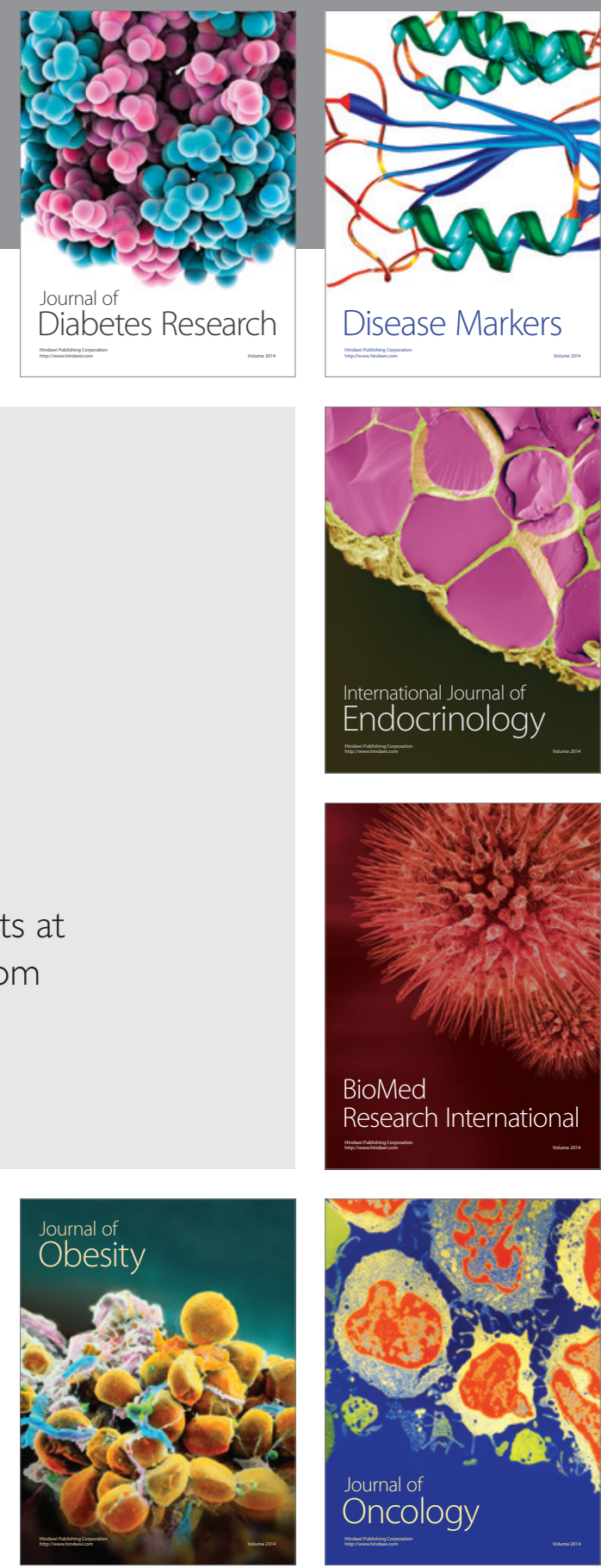

Disease Markers
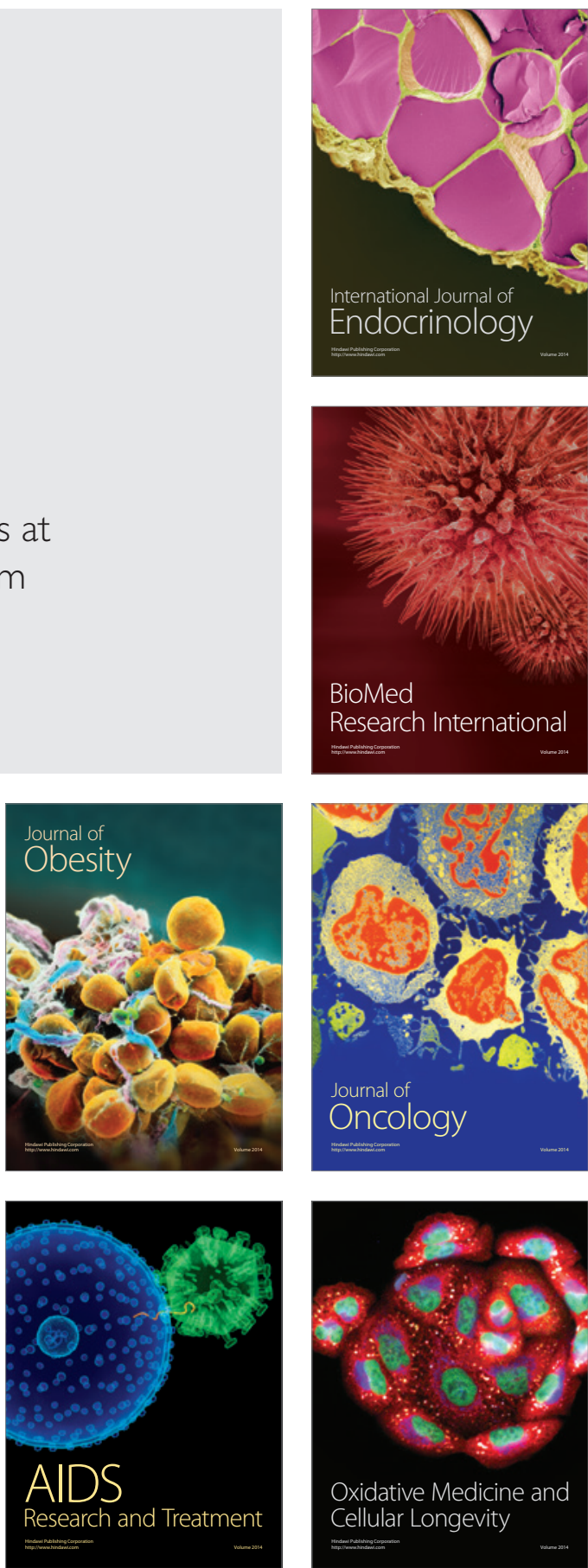